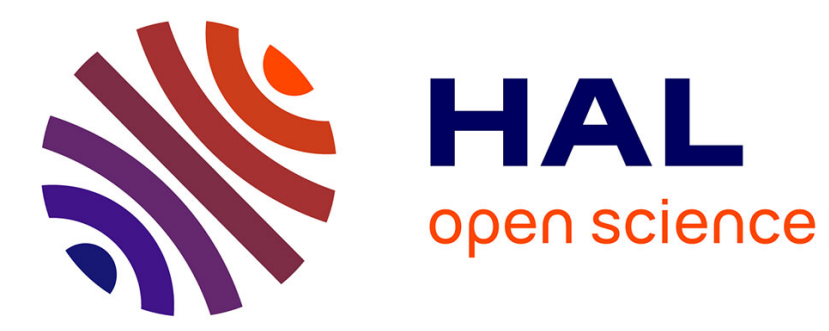

\title{
Relation entre les stéréotypes sexués associés aux pratiques sportives et la motivation autodéterminée des élèves en éducation physique et sportive

\author{
Aïna Chalabaev, Philippe Sarrazin
}

\section{- To cite this version:}

Aïna Chalabaev, Philippe Sarrazin. Relation entre les stéréotypes sexués associés aux pratiques sportives et la motivation autodéterminée des élèves en éducation physique et sportive. Science et Motricité : revue scientifique de l'ACAPS / ACAPS, 2009, 66, pp.61-70. 10.3917/sm.066.0061 . hal00390116

\section{HAL Id: hal-00390116 https://hal.science/hal-00390116}

Submitted on 31 May 2009

HAL is a multi-disciplinary open access archive for the deposit and dissemination of scientific research documents, whether they are published or not. The documents may come from teaching and research institutions in France or abroad, or from public or private research centers.
L'archive ouverte pluridisciplinaire HAL, est destinée au dépôt et à la diffusion de documents scientifiques de niveau recherche, publiés ou non, émanant des établissements d'enseignement et de recherche français ou étrangers, des laboratoires publics ou privés. 
Relation entre les stéréotypes sexués associés aux pratiques sportives et la motivation autodéterminée des élèves en éducation physique et sportive

\author{
Aïna Chalabaev et Philippe Sarrazin \\ Université Joseph Fourier de Grenoble
}

Science et Motricité (2009), 66, 61-70.

La correspondance concernant cet article, doit être addressee à Philippe Sarrazin, Laboratoire Sport et Environnement Social, Université Joseph Fourier - UFRAPS, BP53, 38041 Grenoble cedex 9, France. E-mail: philippe.sarrazin@ujf-grenoble.fr 


\title{
Résumé
}

Cette étude examine (1) les liens entre le stéréotype «masculin » ou «féminin »véhiculé par l'activité sportive et la motivation autodéterminée des élèves en Éducation Physique et Sportive, et (2) le rôle d'un médiateur - la compétence perçue -, et d'un modulateur - le degré d'adhésion au stéréotype -, dans cette relation. Cent-soixante-dix-huit collégiens ont rempli un questionnaire lors d'un cycle de football ou de danse. Les analyses montrent que les élèves sont plus autodéterminés quand ils pratiquent une activité qui véhicule un stéréotype conforme à leur sexe que quand ça n'est pas le cas. Cette relation est médiatisée par la compétence perçue, mais pas modulée par l'adhésion de l'élève au stéréotype. Les résultats sont discutés à la lumière de la théorie de l'autodétermination.

Mots-clés : Stéréotypes sexués ; motivation autodéterminée ; compétence perçue ; médiation ; modulation

\begin{abstract}
This study examines (1) the relationships between the "masculine" or "feminine" stereotype associated with a sport activity and students' self-determined motivation in physical education classes, and (2) the role of a mediator - perceived competence -, and of a moderator - the extent to which students endorse the stereotype - in this relationship. One hundred seventy-eight high school students filled out a questionnaire during a lesson of football or dancing. The analyses showed that students are more self-determined when they are engaged in an activity associated with a stereotype congruent with their sex than when it is not. This relationship is mediated by the perceived competence, but not moderated by the stereotype endorsement. Results are discussed in the light of self-determination theory.
\end{abstract}

Key-words: Sex stereotypes ; self-determined motivation ; perceived competence ; stereotype endorsement ; mediation ; moderation 
L'Education Physique et Sportive (EPS) est une discipline scolaire marquée par d'importants écarts de réussite entre garçons et filles, les premiers y obtenant en moyenne un point de plus que les filles au baccalauréat (Ministère de 1'Education Nationale, de la Recherche et de la Technologie, 2000). Pour expliquer ces différences sexuées, les travaux en psychologie sociale accordent une place centrale aux stéréotypes, qui, dans une approche socio-cognitive, constituent des schémas cognitifs, c'est-à-dire des connaissances abstraites et générales à propos d'une catégorie sociale (e.g., Fiske \& Taylor, 1991). Plusieurs travaux montrent que les Activités Physiques Sportives et Artistiques (APSA) véhiculent des stéréotypes sexués: certaines sont considérées comme plus appropriées aux garçons (e.g., rugby), d'autres aux filles (e.g., danse), même si certaines sont perçues comme pouvant être pratiquées par les deux sexes (e.g., natation) (e.g., Fontayne, Sarrazin, \& Famose, 2001).

Ces stéréotypes sexués pourraient être à l'origine des différences sexuées observées en EPS, en influençant la motivation des élèves. Selon le modèle expectation-valence d'Eccles (e.g., Fredricks \& Eccles, 2005), le choix des individus de pratiquer ou non une activité est principalement déterminé par leurs attentes de succès (i.e., probabilité de réussir une activité) et la valeur subjective de celle-ci (i.e., son degré d'attractivité). Des études montrent en effet que les filles font moins de sport que les garçons parce qu'elles se sentent moins compétentes et lui accordent moins de valeur qu'eux (e.g., Fredricks \& Eccles, 2005). Selon le modèle d'Eccles, ces différences de perceptions résultent de l'internalisation des rôles sexués, qui définissent ce qui est socialement acceptable et attendu de faire pour les hommes et les femmes. Des études ont montré que les individus qui adhèrent aux rôles sociaux masculins tendent à préférer la pratique des APSA masculines et à rejeter les féminines, et ceux qui adhèrent aux rôles sociaux féminins présentent des patrons motivationnels inverses (e.g., Fontayne et al., 2001 ; Fontayne, Sarrazin, \& Famose, 2002 ; Guillet, Sarrazin, Fontayne, \& Brustad, 2006). Par ailleurs, le choix d'une APSA 
Stéréotypes et motivation autodéterminée 4

conforme au rôle sexué internalisé par l'individu semble médiatisé par une compétence perçue et un intérêt élevés pour celle-ci (Fontayne et al., 2002 ; Guillet el al., 2006).

L'objectif principal de cette étude est de prolonger cette ligne de recherche en l'intégrant à une autre théorie particulièrement heuristique pour comprendre les comportements : la théorie de l'autodétermination (TAD ; Deci \& Ryan, 2002 ; voir également Vallerand \& Grouzet, 2001). En effet, si le modèle d'Eccles et collaborateurs et particulièrement approprié pour rendre compte des choix individuels quand une alternative est possible (e.g., quel métier faire ? quelle filière choisir? que activité de loisir sélectionner ?), il est moins adapté pour expliquer l'investissement manifesté dans une activité obligatoire. La TAD, quant à elle, permet cela car elle distingue différents types de motivation en fonction du degré avec lequel le comportement est spontané, autonome et émane d'une force intérieure versus est obligé, contraint et émane d'une force extérieureł Trois formes principales de motivation sont envisagées : la motivation intrinsèque, la motivation extrinsèque, et l'amotivation. La motivation intrinsèque (MI) représente le plus haut degré de motivation autodéterminée et se réfère aux situations dans lesquelles l'individu s'engage librement dans les activités pour le plaisir qu'elles procurent (MI à la stimulation), les défis (MI à l'accomplissement) et les opportunités d'apprentissage (MI à la connaissance) qu'elles engendrent. A 1'inverse, la motivation extrinsèque se réfère aux situations dans lesquelles l'individu s'engage dans une activité pour les conséquences de celle-ci plus que pour l'activité en elle-même, que ce soit (1) afin d'obtenir une récompense ou éviter une punition (i.e., régulation externe), (2) en raison d'un sentiment de culpabilité (i.e., régulation introjectée), (3) la perspective d'atteindre quelque chose d'utile et de valorisé par l'individu (i.e., régulation identifiée) ou (4) pour réaliser quelque chose en cohérence avec les valeurs et les buts de l'individu (i.e., régulation intégrée). Les deux derniers types de régulation sont autodéterminés contrairement aux deux premiers. Enfin, l'amotivation - quand l'individu ne perçoit aucun lien 
Stéréotypes et motivation autodéterminée 5

entre ses actions et les conséquences qu'il pourrait en attendre sur l'environnement - constitue le niveau le plus faible d'autodétermination.

L'intérêt d'intégrer la TAD à l'étude de l'influence des stéréotypes sexués en EPS repose sur les conséquences que la motivation autodéterminée engendre. Plusieurs travaux ont montré qu'une motivation autodéterminée est associée à une plus grande attention, un plaisir plus important, une persistance plus élevée, ou encore à une meilleure performance dans l'activité qu'une motivation non autodéterminée (voir Vallerand \& Grouzet, 2001 pour une revue). La mise en évidence de relations entre les stéréotypes sexués et la motivation autodéterminée impliquerait donc des conséquences importantes des stéréotypes, sur les plans cognitif, affectif et comportemental.

Bien que les liens entre les stéréotypes sexués et la motivation autodéterminée n'ait, à notre connaissance, pas été mis en évidence, cette relation semble tout à fait possible. En effet, selon le modèle hiérarchique de la motivation intrinsèque et extrinsèque de Vallerand (e.g., Vallerand \& Grouzet, 2001), la motivation est largement dépendante de facteurs sociaux, dont les facteurs globaux, omniprésents dans les différents aspects de la vie de la personne. Si l'on considère les stéréotypes sexués comme des facteurs globaux, ceux-ci pourraient affecter la motivation autodéterminée.

Par ailleurs, la TAD postule que l'influence des facteurs sociaux sur la motivation n'est pas directe, mais médiatisée par trois besoins psychologiques - compétence, autonomie et appartenance sociale - que ces facteurs nourrissent ou entravent. Ainsi, les facteurs sociaux qui améliorent la perception de compétence vont, du fait de la satisfaction de ce besoin psychologique, promouvoir une motivation autodéterminée. Sur la base de cette théorie, nous émettons 1'hypothèse d'un lien entre les stéréotypes associés aux APSA et la motivation des garçons et des filles en EPS : celle-ci devrait être moins autodéterminée quand ils ou elles 
pratiquent une activité dont les stéréotypes ne sont pas en adéquation avec leur sexe (e.g., le foot pour les filles et la danse pour les garçons) que dans le cas contraire. D'autre part, ce lien devrait être médiatisé par les perceptions de compétence: garçons et filles devraient se sentir plus compétents quand l'activité est conforme à leur sexe et moins compétents quand celle-ci ne l'est pas. En retour, cette perception de compétence devrait être reliée à une plus grande motivation autodéterminée.

Un autre objectif de cette étude est d'examiner si le degré d'adhésion de l'individu aux stéréotypes sexués module le lien entre le stéréotype associé à une APSA et la motivation pour celle-ci. Plusieurs auteurs ont insisté sur l'importance de distinguer les stéréotypes culturels, véhiculés par une culture particulière, des stéréotypes personnels propres à l'individu (e.g., Devine, 1989). Dans la mesure où ces derniers peuvent différer des premiers, la prise en considération de cette distinction paraît nécessaire. Nous émettons l'hypothèse d'un lien d'autant plus fort entre le stéréotype sexué véhiculé par l'APSA et la motivation autodéterminée pour celle-ci, que l'individu adhère pleinement au stéréotype.

$$
\text { Méthodologie }
$$

\section{Participants et procédure}

Deux cent soixante-cinq élèves (126 filles et 139 garçons) âgés en moyenne de 13.2 ans $(E T=1.31)$ et issus de 14 classes de la $6^{\text {ème }}$ à la $3^{\text {ème }}$ ont participé à 1'étude. Quatre-vingt-neuf élèves ont pris part à un cycle de danse et 176 à un cycle de football ${ }^{1}$. Au début d'une séance se situant au milieu du cycle, les élèves ont rempli un questionnaire mesurant la connaissance du

\footnotetext{
${ }^{1}$ Les analyses ont d'abord été effectuées avec un échantillon de 89 participants à un cycle de football, tiré au hasard de l'échantillon initial, afin d'équilibrer les deux groupes de participants. Dans la mesure où les résultats étaient sensiblement identiques à ceux trouvés avec l'échantillon complet, seuls ces derniers seront donnés.
} 
stéréotype sexué associé à l'activité et leur adhésion à celui-ci, ainsi que leurs perceptions de compétence et motivation autodéterminée pour celle-ci.

Mesures

\section{Motivation autodéterminée pour l'activité}

Une version adaptée pour l'EPS de l'échelle de motivation envers l'éducation (Vallerand, Blais, Brière, et Pelletier, 1989) a été utilisée pour mesurer les différentes formes de motivations des participants. L'amorce de cette échelle était : « Je pratique la danse/le football parce que... ». L'échelle comportait trois items pour chaque type de motivation, à savoir la MI à la stimulation (MIS), la MI à l'accomplissement et à la connaissance ${ }^{2}$ (MIAC), la régulation identifiée (RID), la régulation introjectée (RIJ), la régulation externe (REX), et enfin l'amotivation (AM). Les pratiquants devaient énoncer leur degré d'accord sur une échelle de type Likert allant de (1) «pas du tout d'accord » à (7) « tout à fait d'accord ».

Dans cette étude, chacune des sous-échelles présentant une consistance interne adéquate $(\alpha>.70)$ la moyenne des items de chaque sous-échelle a été calculée. L'indice de motivation autodéterminée a ensuite été calculé en pondérant les scores de chaque sous-échelle selon leur position sur le continuum d'autodétermination (i.e., motivation intrinsèque vs. motivation extrinsèque), selon la formule suivante : [(2×(MIS+MIAC)/2) + RID $]-[((\mathrm{RIJ}+\mathrm{REX}) / 2)+$ $(2 \times A M)]$. Plus cet indice est élevé, plus la motivation du participant est autodéterminée. Cet indice a été largement utilisé dans des études antérieures et présente des caractéristiques psychométriques très satisfaisantes (pour une revue voir Vallerand \& Grouzet, 2001).

${ }^{2}$ Contrairement à l'outil initial, les sous-échelles de motivation intrinsèque à la connaissance et à l'accomplissement ont été fusionnées parce que des entretiens et des analyses préliminaires ont montré que les collégiens ne distinguaient pas les nuances entre ces deux types de motivation. 
Stéréotypes et motivation autodéterminée 8

\section{Perception de compétence}

Une échelle composée de trois items a mesuré la perception de compétence de l'élève (e.g., «j’ai le sentiment que mon niveau en danse/football est : »). Les élèves répondaient sur une échelle en 7 points de (1) très faible à (7) très fort. Cette échelle a déjà été utilisée dans le passé et présente des qualités psychométriques très satisfaisantes (e.g., Marsh, Chanal, \& Sarrazin, 2006). La consistance interne étant bonne dans cette étude $(\alpha=.78)$, la moyenne des items a été calculée.

\section{Adhésion aux stéréotypes sexués associés au football et à la danse}

L'adhésion des élèves aux stéréotypes selon lesquels (1) les garçons sont meilleurs que les filles en football et (2) les filles sont meilleures que les garçons en danse a été mesurée par deux items, élaborés à partir des travaux de Bonnot et Croizet (in press) : «personnellement, je trouve que les filles ont un niveau en danse/football : » et «personnellement, je trouve que les garçons ont un niveau en danse/football : ». Les élèves répondaient sur une échelle de (1) très faible à (7) très élevé. L'indice d'adhésion au stéréotype a été calculé en effectuant la différence entre les deux items. Pour la danse, c'est la différence entre l'item relatif aux filles et l'item relatif aux garçons qui a été calculée. Pour le football, c'est la différence inverse qui a été calculée. Ainsi, plus le score est positif, plus l'adhésion aux stéréotypes est forte.

\section{Perception des stéréotypes sexués associés au football et à la danse}

La perception des stéréotypes relatifs au football et à la danse a été mesurée de la même manière que l'adhésion au stéréotype, à l'exception qu'il était demandé aux élèves d'indiquer leur perception de ce que les gens pensent en général (e.g., « en général, les gens pensent que les filles ont un niveau en danse/football : ») (Bonnot \& Croizet, in press).

\section{Stéréotype sexué véhiculé par les APSA}

Sur la base des travaux antérieurs (e.g., Fontayne et al., 2001), nous avons considéré que le football et la danse véhiculaient respectivement un stéréotype masculin et féminin. 


\section{Résultats}

Des analyses préliminaires ont été effectuées sur les échelles de perception des stéréotypes afin de vérifier si les élèves avaient conscience des stéréotypes sexués attachés aux deux APSA. Deux tests $t$ sur séries appariées ont été réalisés sur l'item «fille» et l'item «garçon ». Les résultats font apparaître une conscience des stéréotypes sexués associés aux deux APSA. En football les scores de l'échelle relative au niveau estimé des garçons par «les gens en général», sont supérieurs à ceux de l'échelle relative au niveau estimé des filles (respectivement $M=5.84$, $E T=1.00$ vs. $M=2.44, E T=1.24), t_{175}=27.38, p<.001$. En danse, les scores de l'échelle relative au niveau estimé des filles sont supérieurs à ceux de l'échelle relative au niveau estimé des garçons (respectivement $M=5.48, E T=1.32$ vs. $M=3.53, E T=1.57$ ), $t_{88}=8.35, p<.001$. Un indice de consensus ${ }^{3}$ représentant le degré d'accord des individus quant à leur perception du stéréotype a également été calculé (James, Demaree, \& Wolf, 1984). Cet indice est de 0.75 et 0.62 pour les items relatifs au niveau des garçons et des filles en football, respectivement, indiquant un accord large des participants sur ces items. Concernant la danse, cet indice est de 0.57 et 0.39 pour les items relatifs au niveau des filles et des garçons respectivement, montrant un degré d'accord large pour le premier item mais faible pour le second.

Dans un second temps, le lien entre le caractère approprié versus non approprié de 1'APSA et la motivation autodéterminée a été examiné, par un test $t$ de Student. Le résultat indique que conformément à notre hypothèse, les élèves pratiquant une activité appropriée à leur sexe avaient une motivation significativement plus autodéterminée $(M=3.19, E T=7.57)$ que

\footnotetext{
${ }^{3} L^{\prime}$ 'indice d'accord $r_{\mathrm{wg}(\mathrm{j})}$ se calcule de la façon suivante $: r_{\mathrm{wg}(\mathrm{j})}=1-\left(S_{\mathrm{xj}}^{2} / \sigma_{\mathrm{EU}}^{2}\right)$, avec $S_{\mathrm{xj}}^{2}$ correspondant à la variance observée de la variable $X$ et $\sigma_{\mathrm{EU}}^{2}$ correspondant à la variance attendue, égale à $\left(A^{2}-1\right) / 12, A$ étant le nombre d'alternatives de réponse dans l'échelle de mesure.
} 
Stéréotypes et motivation autodéterminée 10

ceux pratiquant une activité non appropriée à leur sexe $(M=-3.32, E T=7.80), t_{263}=-6.89, p<$ .001

Dans un troisième temps, nous avons examiné si la perception de compétence médiatisait la relation existant entre le stéréotype sexué véhiculé par l'APSA et la motivation autodéterminée des élèves. Pour cela, nous avons suivi la procédure de Baron et Kenny (1986), qui proposent quatre conditions nécessaires à la mise en évidence d'une médiation : (1) la variable indépendante $X$ (i.e., stéréotype véhiculé par l'APSA) prédit la variable dépendante $Y$ (i.e., motivation autodéterminée) ; (2) $X$ prédit la variable médiatrice $M$ (i.e., perception de compétence) ; (3) $M$ prédit $Y$ lorsqu'on contrôle statistiquement la relation entre $X$ et $Y$; (4) la relation entre $X$ et $Y$ devient non significative (en cas de médiation complète) ou diminue (en cas de médiation partielle) lorsque la relation entre $M$ et $Y$ est préalablement contrôlée.

Afin de réaliser ces analyses, l'activité a été codée (1) quand elle était appropriée au sexe du participant et (-1) dans le cas contraire. La première analyse de régression montre une relation significative entre le caractère approprié ou non de l'activité et la motivation autodéterminée envers l'activité $\left(\beta=.39, p<.001, R^{2}=.15\right)$, confirmant le résultat précédent. La seconde analyse de régression indique une relation significative entre le caractère approprié ou non de l'activité et la perception de compétence $\left(\beta=.22, p<.001, R^{2}=.05\right)$, les élèves se percevant plus compétents dans l'activité appropriée à leur sexe $(M=4.09, E T=0.74)$ que dans 1'autre $(M=3.72, E T=$ 0.90). Enfin, quand la motivation autodéterminée a été régressée sur la variable indépendante et le médiateur, l'analyse s'est révélée significative $F(2,262)=29.33 ; p<.001, R^{2}=.18$, la perception de compétence $(\beta=.18, p<.01)$ prédisant significativement la motivation autodéterminée, et la relation indirecte entre le caractère approprié ou non de l'activité $(\beta=.35, p$ $<.001)$ et la motivation autodéterminée étant plus faible que la relation directe. Pris ensemble, 
Stéréotypes et motivation autodéterminée 11

ces résultats indiquent que le lien entre le caractère approprié ou non de l'activité et la motivation autodéterminée est partiellement médiatisé par la perception de compétence.

Enfin, nous avons examiné si le degré d'adhésion de l'individu aux stéréotypes sociaux modulait le lien qui existe entre le stéréotype sexué véhiculé par une APSA et la motivation pour celle-ci. Tout d'abord, des analyses descriptives montrent que les élèves ont adhéré au stéréotype relatif à la meilleure performance des garçons en football $(M=1.66, E T=2.05)$ et des filles en danse $(M=0.62, E T=2.12)$. Par ailleurs, cette adhésion a été largement partagée, l'indice de consensus étant de 0.78 pour le football et de 0.76 pour la danse. Ensuite, la motivation autodéterminée a été régressée sur le caractère approprié ou non de l'activité, l'adhésion au stéréotype de l'élève que nous avons centrée, et le terme d'interaction entre ces deux variables. L'analyse s'est révélée significative $F(3,261)=17.60 ; p<.001, R^{2}=.17$, le caractère approprié ou non de l'activité $(\beta=.34, p<.001)$ et l'adhésion au stéréotype $(\beta=.12, p=.06)$ prédisant significativement ou de manière marginale la motivation autodéterminée, mais pas le terme d'interaction $(\beta=.02, n s)$. Autrement dit, le lien entre le caractère approprié ou non de l'activité et la motivation autodéterminée n'est pas modulé par l'adhésion au stéréotype, ces deux variables tendant à prédire individuellement et de manière additive la motivation autodéterminée: plus l'activité est appropriée au sexe de l'élève et/ou plus l'élève adhère au stéréotype, plus il est autodéterminé pour l'activité.

\section{Discussion}

Les travaux réalisés dans le cadre de la TAD (e.g., Deci \& Ryan, 2002) montrent que contrairement aux autres, les élèves les plus autodéterminés pour une activité manifestent une grande persévérance en cas de difficulté et obtiennent des performances et des notes plus élevées (e.g., Boiché et al., 2006). Il semble donc important de mieux connaître les antécédents et les processus qui régulent cette motivation. Les objectifs de cette recherche étaient (1) d'étudier le 
Stéréotypes et motivation autodéterminée 12

lien entre le stéréotype sexué véhiculé par le football / la danse et la motivation autodéterminée des élèves envers ces activités dans le cadre de l'EPS, (2) d'examiner le rôle médiateur de la perception de compétence et (3) le rôle modulateur de l'adhésion au stéréotype, dans cette relation.

En accord avec les travaux fondés sur d'autres modèles motivationnels (e.g., Fontayne et al., 2002 ; Guillet et al., 2006), cette étude confirme que les élèves étaient plus autodéterminés pour l'activité quand elle véhiculait un stéréotype conforme à leur sexe que quand ce n'était pas le cas. Par ailleurs, ce lien semble partiellement médiatisé par leur perception de compétence. Autrement dit, quand l'activité est conforme au sexe des participants (i.e., les filles en danse et les garçons en football), ces derniers se sentent plus compétents que dans le cas contraire. En retour, cette perception de compétence est reliée à une plus grande motivation autodéterminée. Néanmoins, le stéréotype sexué de l'activité continuait à prédire la motivation après avoir contrôlé la compétence perçue des élèves. Ce résultat signifie qu'il existe probablement d'autres médiateurs non pris en compte dans cette étude. La TAD apporte des éléments de réponse à cette interrogation. Selon cette théorie, la motivation autodéterminée est également médiatisée par les perceptions d'autonomie (i.e., désir d'être à l'origine de son propre comportement) et d'appartenance sociale (i.e., désir d'être connecté socialement avec des personnes qui nous sont significatives) (e.g., Vallerand \& Grouzet, 2001). Il est possible que le marquage sexuel de l'activité soit relié à la perception d'appartenance sociale. S'investir dans une activité non conforme à son sexe peut entraîner une désapprobation de la part des pairs, et réduire ainsi les perspectives de se sentir connecté avec ces derniers. Des études futures devraient tester cette hypothèse, et de manière plus générale chercher à examiner les différents médiateurs de l'influence du marquage sexuel de l'APSA sur la motivation autodéterminée. 
Stéréotypes et motivation autodéterminée 13

Les résultats montrent par ailleurs que le degré d'adhésion de l'élève au stéréotype ne module pas le lien qui existe entre le stéréotype sexué véhiculé par une APSA et la motivation pour celle-ci. Ce résultat peut paraître surprenant dans la mesure où des études antérieures ont montré que les individus s'engageaient dans des APSA conformes à leur genre. On pouvait donc s'attendre à ce que les élèves qui n'adhèrent pas au stéréotype sexué associé à l'activité non conforme à leur sexe soient moins affectés par le marquage sexuel de ces activités. Nos résultats font apparaître une tendance à un effet simple de l'adhésion au stéréotype. Autrement dit, la conformité de 1'APSA à son sexe et l'adhésion aux stéréotypes entraînent tous deux une plus grande motivation autodéterminée que dans les cas contraires. Cette relation positive entre l'adhésion aux stéréotypes et la motivation autodéterminée, quel que soit le caractère approprié au sexe de l'APSA est inattendue. L'absence d'effet d'interaction pourrait s'expliquer par un degré d'adhésion largement partagé par les élèves, qui conduirait à une distribution déséquilibrée du degré d'adhésion au stéréotype. Les résultats ont montré que c'est effectivement le cas pour le football, cependant, la représentation de la danse en tant que pratique sexuée est moins partagée par les élèves - sans doute parce qu'elle recouvre des pratiques diverses, comme la danse classique ou le hip-hop. Des études futures basées sur un échantillon plus important permettraient d'examiner le rôle modulateur de l'adhésion au stéréotype dans l'influence du marquage sexuel de l'APSA sur la motivation autodéterminée. Il serait intéressant notamment d'examiner les déterminants du degré d'adhésion à un stéréotype. En d'autres termes, pourquoi certains individus adhèrent-ils au stéréotype et d'autres pas? D’une manière générale, comment les stéréotypes se forment-ils et qu'est-ce qui mène celui-ci à être activé dans l'esprit des individus ? Pour aborder ces questions, il serait utile de s'appuyer sur la théorie de l'AutoCatégorisation (Turner, Hogg, Oakes, Reicher, \& Wetherell, 1987), selon laquelle certains contextes sociaux (dont le sport et/ou l'EPS font partie) rendent l'identité sexuelle saillante au plan cognitif, activant 
Stéréotypes et motivation autodéterminée 14

ainsi des processus de dépersonnalisation des individus et les stéréotypes sexués associés aux pratiques sportives. L'examen de ces questions permettrait de mieux comprendre l'influence des stéréotypes dans la motivation en EPS et les moyens de la réduire.

\section{Références}

Ashmore, R.D., \& De1 Boca, F.K. (1981). Conceptual approaches to stereotypes and stereotyping. In D.L. Hamilton (Ed.), Cognitive processes in stereotyping and intergroup behavior (pp. 1-35). Hillsdale, NJ : Lawrence Erlbaum.

Baron, R.M., \& Kenny, D.A. (1986). The moderator-mediator variable distinction in social psychological research: Conceptual, strategic, and statistical considerations. Journal of Personality and Social Psychology, 51, 1173-1182.

Bem, S.L. (1981). Gender schema theory: A cognitive account of sex-typing. Psychological Review, 88, 354-364.

Bonnot, V., \& Croizet, J.-C. (in press). Stereotype internalization and women's math performance: The role of interference in working memory. Journal of Experimental Social Psychology.

Deci, E.L., \& Ryan, R.M. (Eds.) (2002). Handbook of Self-determination research. Rochester: The University of Rochester Press.

Devine, P. G. (1989). Stereotypes and prejudice: Their automatic and controlled components. Journal of Personality and Social Psychology, 56, 5-18.

Fiske, S. T., \& Taylor, S. E. (1991). Social Cognition (2 ${ }^{\text {nd }}$ ed.). New York: McGraw-Hill.

Fontayne, P., \& Sarrazin, P. (2001) Genre et motivation dans le domaine du sport. In F. Cury, et P. Sarrazin (Eds.). Théories de la motivation et pratiques sportives : Etat des recherches (pp. 277-295). Paris, PUF, pratiques corporelles. 
Stéréotypes et motivation autodéterminée 15

Fontayne, P., Sarrazin, P., \& Famose, J.-P. (2001). Les pratiques sportives des adolescents : une différenciation selon le genre. STAPS, 55, 23-37.

Fontayne, P., Sarrazin, P., \& Famose, J.-P. (2002). Effet du genre sur le choix et le rejet des activités physiques et sportives en Education Physique et Sportive : Une approche additive et différentielle du modèle de l'androgynie. Science et Motricité, 45, 45-66.

Fredricks, J.A., \& Eccles, J.S. (2005). Family socialization, gender, and sport motivation and involvement. Journal of Sport \& Exercise Psychology, 27, 3-31.

Guillet, E., Sarrazin, P., Fontayne, P., \& Brustad, R. (2006). Understanding Female Sport Attrition in a Stereotypical Male Sport Within the Framework of Eccles' Expectancy-Value Model. Psychology of Women Quarterly, 30, 358-368.

James, L.R., Demaree, R.G., \& Wolf, G. (1984). Estimating within-group interrater reliability with and without response bias. Journal of Applied Psychology, 69, 85-98.

Marsh, H., Chanal, J., \& Sarrazin, P. (2006). Self-Belief Does Make A Difference: A Reciprocal Effects Model of the Causal Ordering of Physical Self-concept and Gymnastics Performance. Journal of Sports Sciences, 24, 101-111.

Ministère de 1'Education Nationale, de la Recherche et de la Technologie (2000). Les Epreuves du Baccalauréat en Education Physique et Sportive. Rapport de 1'Inspection Générale. Disponible à l'adresse ftp://rf.education.gouv.fr/pub/edutel/syst/igen/baceps.pdf

Turner, J.C., Hogg, M.A., Oakes, P.J., Reicher, S.D., \& Wetherell, M.S. (1987). Rediscovering the social group: A self-categorization theory. Oxford, UK: Blackwell.

Vallerand, R.J., Blais, M.R., Brière, N.M., \& Pelletier, L.G. (1989). Construction et validation de l'échelle de motivation en éducation. Canada Journal of Behavioral Science, 21(3), 323349. 
Stéréotypes et motivation autodéterminée 16

Vallerand, R.J., \& Grouzet, F.M.E. (2001). Pour un modèle hiérarchique de la motivation intrinsèque et extrinsèque dans les pratiques sportives et l'activité physique. In F. Cury et $\mathrm{P}$. Sarrazin (Eds.), Théories de la motivation et pratiques sportives : états de la recherche (pp. 57-95), Paris : PUF. 